\title{
Aesthetic appreciation: event-related field and time-frequency analyses
}

\author{
Enric Munar ${ }^{1}{ }^{*}$, Marcos Nadal $^{1}$, Nazareth P. Castellanos ${ }^{2}$, Albert Flexas ${ }^{1}$, Fernando Maestú ${ }^{2}$, \\ Claudio Mirasso ${ }^{3}$ and Camilo J. Cela-Conde ${ }^{1}$ \\ ${ }^{1}$ Human Evolution and Cognition (EvoCog), University of the Balearic Islands, Palma (Mallorca), Spain \\ ${ }^{2}$ Laboratory of Cognitive and Computational Neuroscience, Complutense University of Madrid-Polytechnic University of Madrid, Madrid, Spain \\ ${ }^{3}$ IFISC, Instituto de Física Interdisciplinar y Sistemas Complejos (CSIC-UIB), University of the Balearic Islands, Palma (Mallorca), Spain
}

Edited by:

Luis M. Martinez, Universidad

Miguel Hernández, Spain

Reviewed by:

Rodrigo Q. Quiroga, University of

Leicester, UK

Manuel Molano, Instituto de

Neurociencias de Alicante, Spain

*Correspondence:

Enric Munar, Human Evolution and

Cognition (EvoCog), University of

the Balearic Islands, Ctra. de

Valldemossa, km. 7'5.,

07122-Palma, Illes Balears, Spain.

e-mail: enric.munar@uib.cat
Improvements in neuroimaging methods have afforded significant advances in our knowledge of the cognitive and neural foundations of aesthetic appreciation. We used magnetoencephalography (MEG) to register brain activity while participants decided about the beauty of visual stimuli. The data were analyzed with event-related field (ERF) and Time-Frequency (TF) procedures. ERFs revealed no significant differences between brain activity related with stimuli rated as "beautiful" and "not beautiful." TF analysis showed clear differences between both conditions $400 \mathrm{~ms}$ after stimulus onset. Oscillatory power was greater for stimuli rated as "beautiful" than those regarded as "not beautiful" in the four frequency bands (theta, alpha, beta, and gamma). These results are interpreted in the frame of synchronization studies.

Keywords: neuroaesthetics, experimental aesthetics, MEG, ERF, visual perception, time-frequency analysis

\section{INTRODUCTION}

Neuroaesthetics is growing fast, and the neural correlates of aesthetic appreciation are now coming into focus (Chatterjee, 2011; Nadal and Pearce, 2011). Neuroimaging studies have revealed that positive aesthetic experiences, reported as high liking, preference, or beauty ratings, are associated with at least three patterns of brain activity. First, the enhancement of low-and high-level visual, somatosensory, and auditory cortical processing has been observed while people report aesthetically positive engagements with paintings or landscape photographs Vartanian and Goel (2004) (Vartanian and Goel, 2004; Yue et al., 2007; Cela-Conde et al., 2009; Cupchik et al., 2009), dance movements or postures (Calvo-Merino et al., 2008, 2010), and music excerpts (Brown et al., 2004; Koelsch et al., 2006), respectively. Second, activity in cortical regions involved in top-down processing and evaluative judgment is also a common finding (Cela-Conde et al., 2004; Jacobsen et al., 2006; Lengger et al., 2007; Cupchik et al., 2009). Finally, several studies have reported activation of cortical and subcortical brain regions considered to be part of the reward circuit. These regions are related with different facets of affective and emotional processing. Namely, the orbitofrontal cortex, which seems to be involved in the representation of reward value, has been associated with positive aesthetic experiences of music (Blood et al., 1999; Blood and Zatorre, 2001), architecture (Kirk et al., 2009a), and paintings (Kawabata and Zeki, 2004; Cupchik et al., 2009; Kirk et al., 2009b). Activity in the anterior cingulate cortex, possibly related with the monitoring of one's own affective state, has also been identified while rating paintings (Vartanian and Goel, 2004; Cupchik et al., 2009), architecture (Kirk et al., 2009a) and music (Blood et al., 1999). Subcortical components of the reward circuit, such as the ventral striatum, the caudate nucleus, the substantia nigra, or the amygdala, have been shown to be involved in aesthetic experiences by a considerable number of studies (Blood et al., 1999; Blood and Zatorre, 2001; Brown et al., 2004; Vartanian and Goel, 2004; Koelsch et al., 2006; Bar and Neta, 2007; Gosselin et al., 2007; Mitterschiffthaler et al., 2007; Cupchik et al., 2009; Kirk et al., 2009b; Salimpoor et al., 2011).

Although these studies collectively provide an overall picture of the brain regions involved in aesthetic appreciation, little is known about the temporal course of the underlying neural processes. In the present study we apply novel neuroimaging data analyses, currently used in diverse areas of the neurosciences, to explore and tentatively characterize the dynamics of the neural correlates of aesthetic preference. We thus aim to overcome a common objection faced by neuroimaging data analysis: the assumption of the stationary nature of neurophysiological signals. Most spectral studies of continuous time series, such as Electroencephalography (EEG) or Magnetoencephalography (MEG) recording, involve the use of a spectral analysis based on Fourier transformation. Although this technique has been extremely fruitful in the advance of neuroscience, it assumes that neural activity under study is stationary, and thus does not allow inferences on dynamical changes. Indeed, any analysis based entirely on the classical Fourier transform ignores the dynamical aspects. New methods suited to reveal temporal variations are therefore, required to study the essential role of temporal resolution.

There have been successful attempts to adapt Fourier-based methods, for example, by means of sliding windows (Bayram and Baraniuk, 1996; Lovett and Ropella, 1997; Xu et al., 1999), similarly to the classical Gabor transform (Mallat, 1999). The wavelet transform is a method of time series analysis capable of coping with complex non-stationary signals - it was, in fact, designed to do just that. Although it has been increasingly used in the 
field of neuroscience during the last decade, it has been part of brain signal analysis from the very beginning. The analysis of EEG recordings has been its most frequent application (see e.g., Alegre et al., 2003; Quiroga and Garcia, 2003; Castellanos and Makarov, 2006; Campo et al., 2010; Castellanos et al., 2010). The wavelet transform technique provides high temporal resolution with good frequency resolution, and offers a reasonable compromise between these parameters. These advantages fit well with the purpose of Time-Frequency (TF) estimation of a signal, allowing the study of the spectral power dynamics, and hence a detailed comparison between experimental conditions during all the steps composing a designed task (Lindsen et al., 2010).

In this article, we report the results of two different analyses of MEG data recorded during a typical aesthetic appreciation task. First, we present the results of a standard event-related field (ERF) analysis. Second, we also performed a TF analysis, which, on the one hand, compared pre- and post-stimulus activity in different frequency bands and, on the other, compared the different activity related with stimuli regarded as beautiful and not beautiful in different bands and brain regions. This kind of TF analysis avoids the misleading simplification resulting from the localization of apparently static and isolated foci of neural activity. It has the potential, therefore, of making a significant step forward in the characterization of the dynamics of large-scale neural communication inherent to aesthetic appreciation, among many other complex cognitive faculties (Lindsen et al., 2010), which emerges from multifaceted cognitive processes related with neural activity in different brain structures and at different time frames.

\section{MATERIALS AND METHODS PARTICIPANTS}

Ten women and 10 men volunteered to perform the aesthetic appreciation task. They were all graduate students at the Complutense University in Madrid. They all had normal or corrected-to-normal vision, and no previous training in art. All were right-handed and gave informed consent. The experiment was approved but the Ethical Committee of the Comunitat Autònoma de les Illes Balears (Spain).

\section{PROCEDURE}

The resolution, size, perceived complexity, color spectrum, and luminous emittance were homogenized for the 400 stimuli used in this study. These five operations and the stimuli were described in detail by Cela-Conde et al. (2009). These images belonged to five different categories, and represented a broad enough range of contents and styles to provide each participant with positive and negative aesthetic experiences throughout the task: (1) Fifty reproductions of abstract paintings; (2) Fifty reproductions of seventeenth and eighteenth century realist paintings; (3) Fifty reproductions of Impressionist paintings; (4) Fifty reproductions of Post-impressionist paintings; (5) Two hundred photographs of landscapes, artifacts, urban scenes, and the like (true-life pictures from the Master Clips Premium Image Collection, IMSI, San Rafael, CA; the book Boring Postcards, London, Phaidon Press; and photographs taken by us). We used the collection Movements in Modern Art from the Tate Gallery, London, as a guide to select artistic styles, to which we added seventeenth and eighteenth century realist painting. To avoid the activation of facial-recognition brain mechanisms, pictures containing close views of humans were not included. Four stimuli (two artistic and two natural) were used for the participants' preliminary training.

Before going into the MEG isolated room, participants received a short briefing about the technique and the aesthetic appreciation task they were required to carry out. They were informed that it was a two-alternative forced choice (2AFC), with two response levels: (a) beautiful, and (b) not beautiful. Participants were asked to indicate whether they found each stimulus to be "beautiful" or "not beautiful", based on their own subjective opinion. Half of them indicated "beautiful" by raising an index finger, and the other half indicated "not beautiful" also by raising an index finger. The remaining stimuli were classified as belonging to the other condition—as "not beautiful" and "beautiful," respectively. Half of the participants used the right index finger to answer and the other half used the left index finger. At the beginning of the task, a gray screen appeared for $1 \mathrm{~s}$, then a stimulus was presented for $3 \mathrm{~s}$, during which the participant could choose to respond or not. There was a random interstimulur interval lasting between 1000 and $1200 \mathrm{~ms}$ after each $3 \mathrm{~s}$ presentation. The same four stimuli were presented at the beginning for all participants' training trials.

During the experimental session, the stimuli were presented using a computer running the SuperLab application. The images were projected with an LCD video projector placed outside of the MEG shielded room onto a series of mirrors located inside, the last of which was suspended $\approx 1 \mathrm{~m}$ above the participant's face. The pictures subtended $1.8^{\circ}$ and $3^{\circ}$ of vertical and horizontal visual angles, respectively. While participants carried out the aesthetic appreciation task, MEG recordings were performed with a whole-head neuromagnetometer (Magnes $2500 \mathrm{WH}, 4-\mathrm{D}$ Neuroimaging) consisting of 148 magnetometer coils.

Raw data were collected using a sampling rate of $668.45 \mathrm{~Hz}$ and band pass filtered between 0.1 and $50 \mathrm{~Hz}$. MEG data were subjected to an interactive environmental noise reduction procedure. Fields were measured during a no task opened-eyes condition. Time-segments containing eye movement or blinks (as indicated by peak-to-peak amplitudes in the electro-oculogram channels in excess of $50 \mu \mathrm{V}$ ) or other myogenic or mechanical artifact were rejected and time windows not containing artifact were visually selected by an experienced investigator, leading to $12 \mathrm{~s}$ clean segments. The minimum number of trials obtained after artifact rejection was 90 for every participant. Digitized MEG data were imported into MATLAB Version 7.4 (Mathworks, Natick, MA) for analysis with custom-written scripts.

\section{DATA ANALYSIS AND RESULTS BEHAVIORAL ANALYSIS}

In order to detect any possible effect of stimuli category on participants' responses, we carried out a means comparison with the "beautiful" responses as dependent variable and stimuli category as independent variable. On average, artistic stimuli were rated as beautiful on 99.7 of the 200 possible occasions. Nonartistic stimuli, on the other hand, were rated as beautiful on 96.1 of the instances. This difference, however, was non-significant $[t(19)=0.69 ; p=0.4]$. 
With regards to the set of artistic stimuli, a one-way (ANOVA) was performed taking into account the four subcategories (abstract, realist, impressionist, and post-impressionist artworks). On average, participants rated 23.4 of the abstract artworks, 24.75 of realist artworks, 26.05 of the impressionist artworks, and 24.85 of the post-impressionist artworks as beautiful. Again, there were no significant differences among the ratings for these stimuli categories, $[F(3,76)=1.693 ; p=0.176]$.

\section{EVENT-RELATED FIELDS (ERF)}

ERFs were derived by averaging single trials. Average ERFs were calculated for each condition (beautiful and not beautiful), individual sensor (148), and participant (20). A period of $500 \mathrm{~ms}$ prior to target onset was defined as the baseline. ERFs were calculated for $100 \mathrm{~ms}$ periods, individual sensor and for each condition (Figure 1). A strong positive effect appeared in the right anterior temporal region within the 100-200 ms window. Its corresponding negative effect appeared in the contralateral hemisphere, this is, the left anterior temporal region. This effect was sustained during the subsequent time windows until $500 \mathrm{~ms}$. This was observed both for beautiful stimuli (Figure 1A) and not beautiful stimuli (Figure 1B).

We selected the main contributing sensors to this effect: sensors $108,109,110,111,126,127,128,129,144,145,146,147$, and
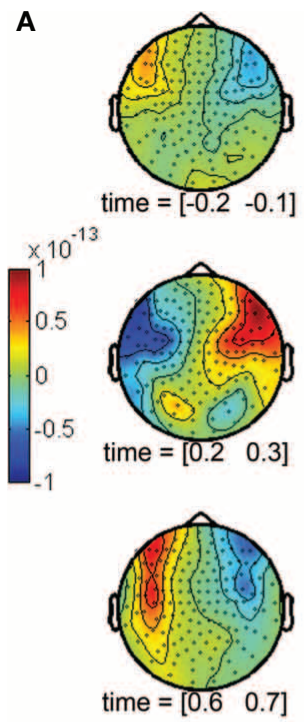

B

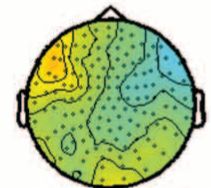

time $=\left[\begin{array}{ll}-0.2 & -0.1\end{array}\right]$
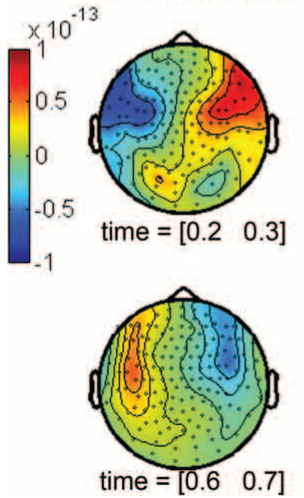
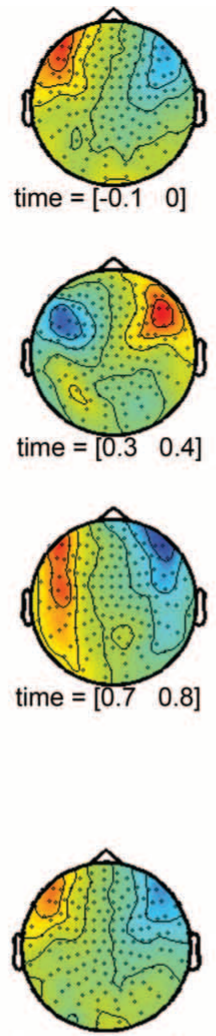

time $=\left[\begin{array}{ll}-0.1 & 0\end{array}\right]$
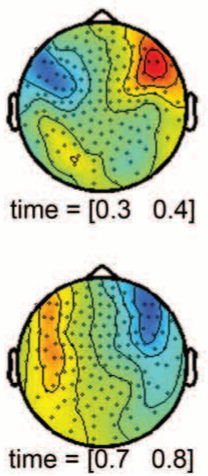
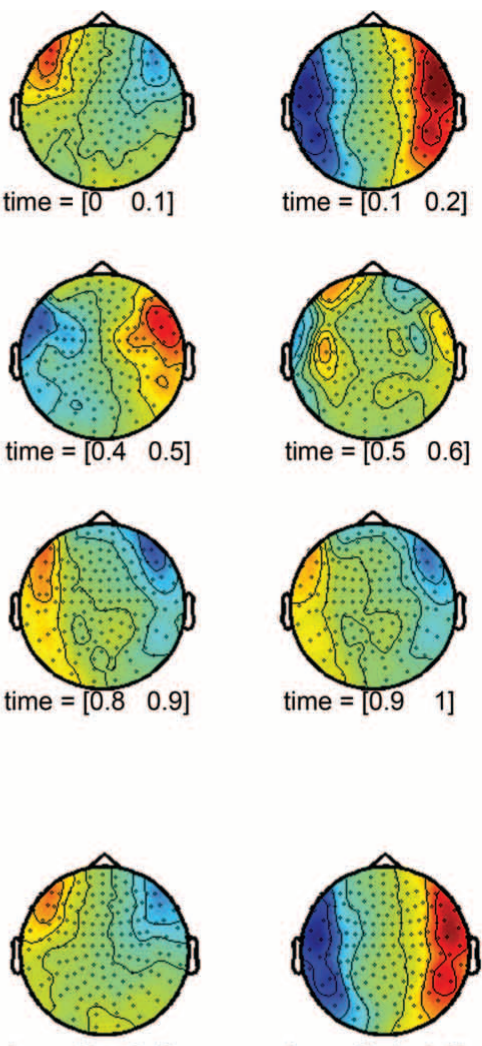

time $=\left[\begin{array}{ll}0 & 0.1\end{array}\right]$

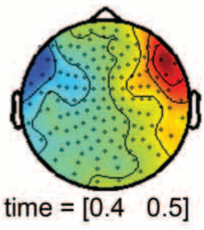

time $=\left[\begin{array}{ll}0.1 & 0.2\end{array}\right]$
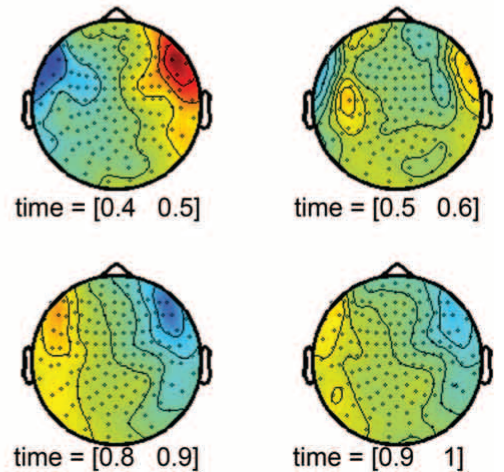

FIGURE 1 | Topographic representations of the activity in 100 ms windows from $\mathbf{2 0 0}$ ms prior to stimulus onset to $1 \mathrm{~s}$ after stimulus onset. (A) Beautiful stimuli. (B) Not beautiful stimuli. 
148 for the right hemisphere; sensors $96,97,98,99,114,115,116$, $117,132,133$, and 134 for the left hemisphere. Grand averages of these sensors were calculated from $0.5 \mathrm{~s}$ prior to stimulus onset to $1 \mathrm{~s}$ after stimulus onset (Figure 2) for both conditions. Three peaks showed the dominant effects during the aesthetic appreciation task in the pointwise ERF analysis: an early component (160-180 ms) with the largest amplitude, an intermediate component (250-280 ms), and a late component (450-480 ms). The last two peaks seemed to be a consequence of the first one, a sort of sustainment of activity.

For statistical analyses, a procedure used in other kinds of ERF and Event-Related Potentials (ERPs) studies was applied to analyze the activity of the MEG waveform, in this case, as a function of rated beauty. Parametric and non-parametric tests were calculated for each time point after stimuli onset for each individual MEG sensor in order to identify the modulation of the ERF as a function of beauty. These analyses were conducted using a significance criterion of $p<0.05$ with Bonferroni correction based on the 1356 tests per analyzed sensor, a test for each epoch. The analysis was performed independently for each sensor. There were no significant differences between beautiful and not beautiful stimuli in the modulation of the ERF.

\section{TIME-FREQUENCY ANALYSIS}

This analysis aimed to overcome the aforementioned criticism of Fourier transformation-based spectral analysis: the assumption of the stationary nature of neurophysiological signals. The wavelet transform's advantages fit well with the purpose of offering a TF characterization of a signal, allowing the study of the spectral

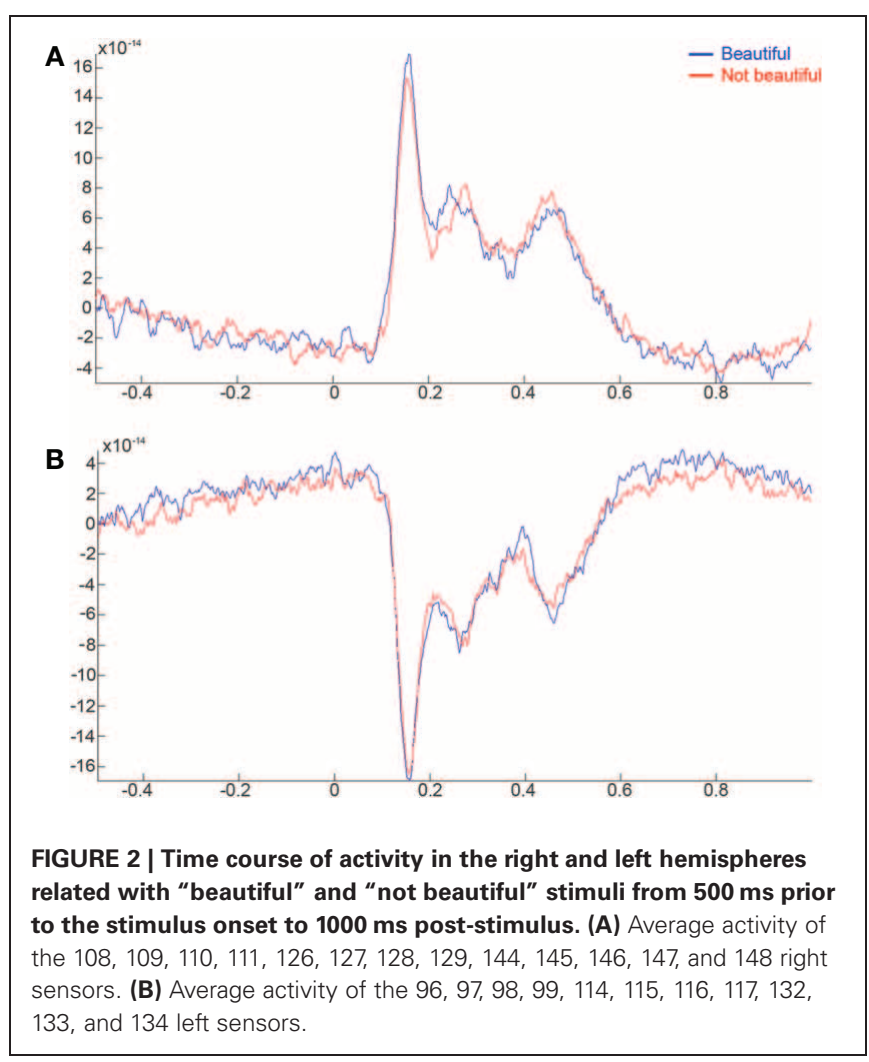

power dynamics and, therefore, a detailed comparison between experimental conditions, i.e., beautiful and not beautiful, during the stages of the aesthetic appreciation task. The wavelet coefficients, $W(p, z)$, can be obtained as follows:

$$
W(p, z)=\frac{1}{\sqrt{p}} \int_{-\infty}^{\infty} x(t) \Psi^{*}\left(\frac{t-z}{p}\right) \mathrm{dt}
$$

where the parameter $z$ defines the time localization, and $p$ the wavelet timescale representing the period of the rhythmic component (Mallat, 1999; Torrence and Compo, 1998; Grinsted et al., 2004). TF representation of MEG data was calculated on a single trial basis for a $1500 \mathrm{~ms}$ time window starting from $500 \mathrm{~ms}$ before and ending $1000 \mathrm{~ms}$ after the onset of the stimulus presentation, using a Morlet wavelet function. Thus, a baseline correction was performed in order to estimate stimulus evoked oscillations. Power in the standard frequency bands of theta $(4-8 \mathrm{~Hz})$, alpha $(8-12 \mathrm{~Hz})$, beta $(12-30 \mathrm{~Hz})$, and gamma $(30-50 \mathrm{~Hz})$ was computed. The sensors were grouped such that they related to five brain regions: Frontal (17 sensors), Right Temporal (30), Left temporal (36), Occipital (32), and Central (33) (Figure 3).

TF sensor representation in the four spectral bands and time were compared between the previous and the posterior activity to the stimulus onset using a Kruskal Wallis test $(p<0.001)$ with a False Discovery Rate (FDR) correction. We used an FDR correction due to the exploratory character of this study, and not an Family wise Error Rate (FWER), which is better suited for confirmatory designs (Storey, 2002; Groppe et al., 2011). Nevertheless, some studies have demonstrated that the FDR control is useful both with "independent test statistics" and "dependent test statistics" (Benjamini and Yekutieli, 2001; Verhoeven et al., 2005) and has been applied to TF analyses in the context of clinical neuroimaging studies (Kobayashi et al., 2009). Note that we refer to time-spectra changes and not TF changes due to the fact that we have averaged frequency in order to estimate the dynamical (time dependent) changes per spectral band and not per frequency scale.

Statistical differences are summarized and represented in Figure 4. Stimuli rated by participants as beautiful and not beautiful were considered separately. The results for the theta band are presented in the upper part of the figure, next the results for the alpha band, and thereafter those for beta and gamma bands. Within each of the bands, the results from the frontal sensors (F) are presented first, then those from the right temporal sensors (RT), then those from the left temporal sensors (LT), followed by those from the occipital sensors $(\mathrm{O})$, and finally those from the central sensors $(\mathrm{C})$. The resulting patterns for stimuli rated as beautiful and not beautiful were very similar. The largest activity in both conditions appeared in the alpha waveband in temporal lobe regions at close to $200 \mathrm{~ms}$ after stimulus onset. These results confirmed the previous ones obtained through ERF analysis, that is to say, there is a maximum activation between 100 and $200 \mathrm{~ms}$, and, in addition, they specify that such activity is characterized by oscillations mainly within the alpha band.

On the other hand, the power values were also statistically analyzed using a Kruskal Wallis test with an FDR correction to compare the spatial-TF patterns between the beautiful and not 


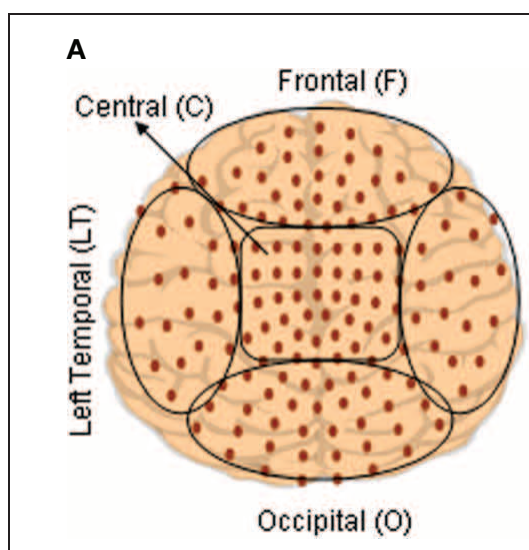

B

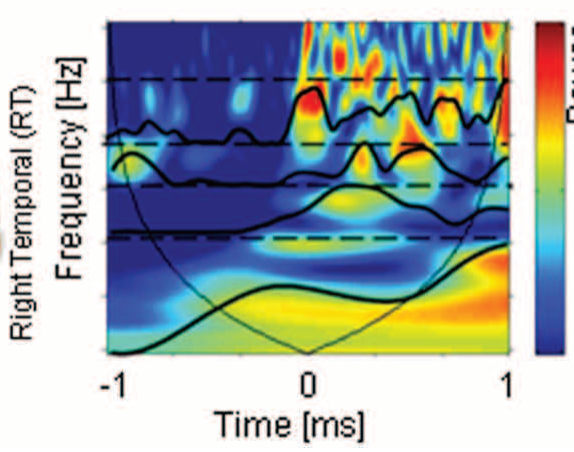

C

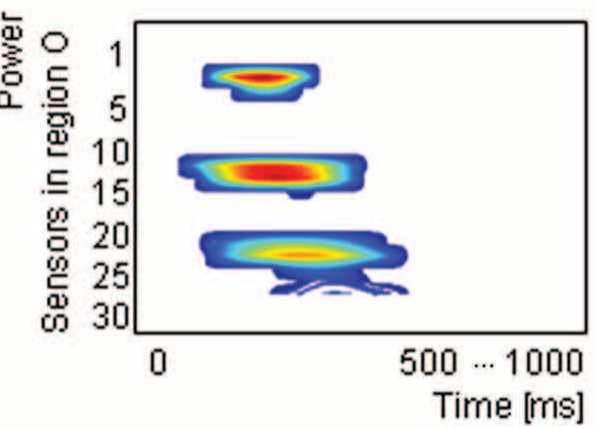

FIGURE 3 | (A) The five defined brain regions: Frontal (F), Central (C), Occipital (O), and Left and Right Temporal (LT, RT) areas.

(B) Time-Frequency representation of one sensor from $1 \mathrm{~s}$ before to $1 \mathrm{~s}$ after stimulus onset. Frequency scales are averaged in standard spectral bands (dash lines). (C) The brain region time-spectral band diagram represents the time dependent spectral changes for all channels belonging to one brain region in a certain spectral band. beautiful stimuli. In this case, there were many more differences, and more intense, between beautiful and not beautiful than the reverse-i.e., not beautiful minus beautiful (Figure 5). For this reason, we present the results of the subtraction of the not beautiful stimuli power from the beautiful stimuli power with a $p<$ 0.0005 - a possible false positive each 2000 contrasts - and the opposite results with a $p<0.05$ - a possible false positive each 20 contrasts.

The most outstanding differences between the beautiful and not beautiful conditions occurred from around $400 \mathrm{~ms}$ onwards. In the theta band, there was a notable power increase in the frontal and left temporal lobe regions with beautiful stimuli compared to not beautiful stimuli. In the same direction, there were noticeable differences in activity in the frontal, occipital and, to a lesser extent, left temporal regions in the alpha band. Although with lesser intensity, significant differences also appeared in the occipital, frontal, left temporal, and right temporal regions in the beta band. Finally, there were also lesser significant differences in the occipital, right temporal, and frontal regions in gamma band.

Importantly, the subtraction not beautiful minus beautiful hardly produced any significant differences (right-hand column of Figure 5), even accepting a very high Type I error $(p<0.05)$. This suggests that after $300-400 \mathrm{~ms}$ the power of the spatial-TF patterns while viewing stimuli rated as beautiful are larger than while viewing stimuli rated as not beautiful in any frequency band and in all cortical regions.

\section{DISCUSSION}

In this study we explored the dynamics of neural activity underlying aesthetic appreciation in two different ways. Our ERF analysis revealed no differences between brain activity related with stimuli rated as beautiful and not beautiful, although both conditions showed a clear peak $170 \mathrm{~ms}$ after stimulus onset. Conversely, TF analysis showed that $300 \mathrm{~ms}$ after stimulus onset activity in the four frequency bands and in the five defined brain areas was greater for stimuli rated as beautiful than as not beautiful.

The brain region labels used to describe profiles of power could be subjected to small spatial deviations. A direct relation between the position of the sensor and the immediate brain region cannot, therefore, be established. However, we have grouped the signals in the sensor space into five sensor groups (F, RT, LT, O, and C) to limit this effect. In addition, the magnetic field measured with MEG is much less distorted by biological tissue than the electric potentials from EEG and, as a result, a much more direct relation between the original source and the signal captured at the sensor space can be expected.

\section{EVENT RELATED FIELDS}

Neuroimaging and neurophysiological techniques have only recently and scantly been used to characterize the dynamical nature of brain activity related with the appreciation of beauty and other aesthetic features. Although such studies are still considerably outnumbered by fMRI experiments designed to identify the spatial location of activity, Jacobsen and Höfel (2003) and de Tommaso et al. (2008) pioneering work constitutes essential reference points for our findings.

In contrast with our ERF results, Jacobsen and Höfel (2003) and de Tommaso et al. (2008) obtained significant EEG differences between activity associated with beautiful and not beautiful stimuli. Jacobsen and Höfel (2003) reported that stimuli regarded as not beautiful were accompanied by a fronto-central negative deflection about $300 \mathrm{~ms}$ after onset. On the other hand, de Tommaso et al. (2008) found an increase in the N2m (260 ms) while participants viewed neutral pictures, unlike the beautiful ones. Interestingly, such N2m amplitude pattern was not observed in a second task where participants were required to perform a simple recognition task with items they had previously rated.

Given that there is no simple correspondence between ERFs and ERPs responses, our results may not be straightforwardly comparable with Jacobsen and Höfel (2003) and de Tommaso et al. (2008). On the one hand, ERFs are sensitive to only of a subset of the neural activity that can be detected by ERPs and, on the other, selectivities that are clear in the ERFs may be diluted with ERPs (Liu et al., 2002). Besides the neuroimaging technique, there were other differences between our study and the other two that may contribute to the different results. Jacobsen and Höfel (2003) 


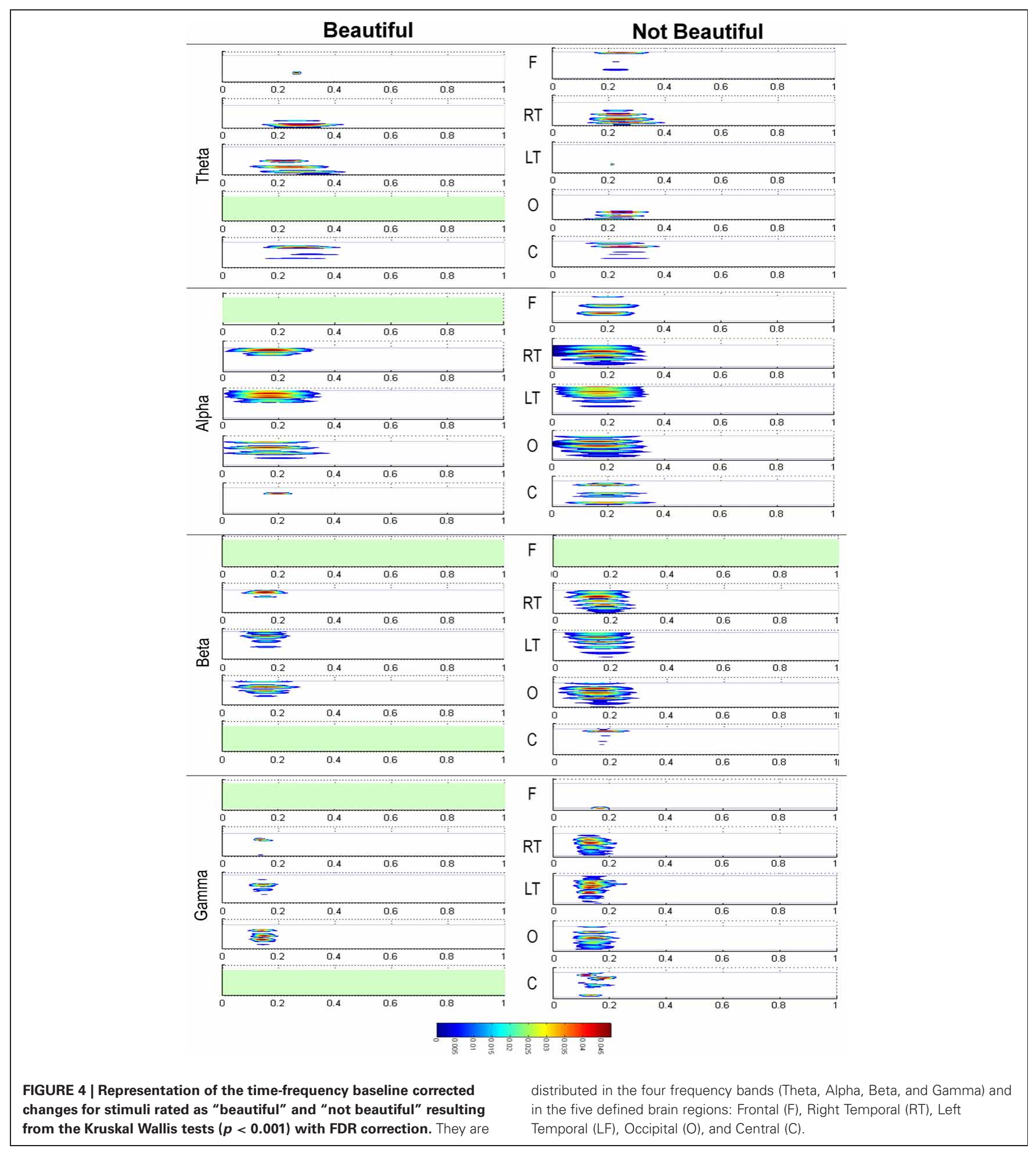

used black and white geometrical stimuli and compared participants' rating of an aesthetic feature (beauty) and a formal feature (symmetry). de Tommaso et al. (2008) used famous paintings and complex colored geometrical shapes, the stimulus presentation lasted $750 \mathrm{~ms}$, and responses were collected on a 10-point scale. We have argued elsewhere that this kind of experimental design and implementation peculiarities can be a source of disparate results (Nadal et al., 2008).

Our ERF analysis showed a peak at $170 \mathrm{~ms}$ followed by prolonged activity with two lesser peaks: 270 and $450 \mathrm{~ms}$. The positive pole of the first component was located in the right temporal region, and the negative one in the contralateral left 


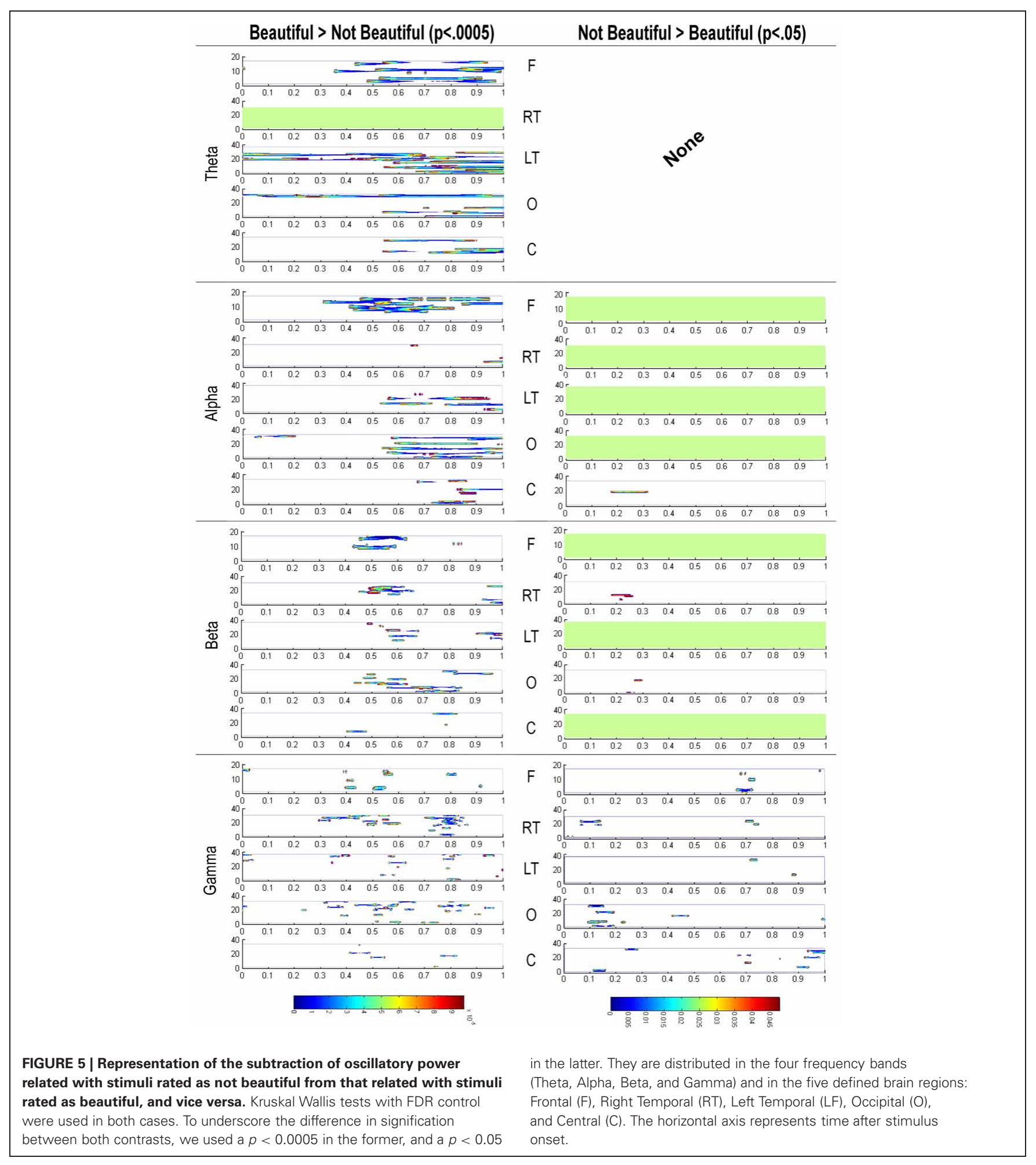

region. The two other components were located more frontally than the first, but equally symmetrical. Previous studies have established associations between early ERF components, approximately at $170 \mathrm{~ms}$, and specific cognitive processes. They have mostly related early ERFs with face processing or emotions, sometimes separately, sometimes jointly.

Most recent studies of facial processing that used ERFs have reported two early MEG responses (Halgren et al., 2000; Liu et al., 2002; Itier et al., 2006; Susac et al., 2010). The first one takes place around $100 \mathrm{~ms}$ and is basically circumscribed to the occipital lobe. Although it is face-selective, it does not appear to be related with facial identification. The M100 is usually considered as indexing 
the early encoding stage of visual information (Itier et al., 2006). The second ERF component related with face processing is M170. The main source is the inferior temporal area, consistent with the fusiform gyrus (Lu et al., 1991; Linkenkaer-Hansen et al., 1998; Halgren et al., 2000; Liu et al., 2000; Taylor et al., 2001; Kloth et al., 2006). Usually, it is of positive polarity on the left hemisphere and negative polarity on the right hemisphere, the opposite pattern to our findings. The M170 is involved in face identification, so its pattern is different depending on individual facial traits. It seems to reflect refined stages where identity encoding begins (Itier and Taylor, 2002; Itier et al., 2006), sensory input is transformed for future processing, sensory code is translated to cognitive processing (Halgren et al., 2000), or/and a deeper processing is carried out as a function of stimulus ambiguity (Itier et al., 2006). Liu et al. (2002) believe that the relation between M100 and M170 might be a continuum along which facial processing becomes increasingly precise.

Although studies examining the early components of neural processing of emotion with ERP analysis abound in the literature, only a few experiments have dealt with the relation between emotional processes and early MEG components-see Olofsson et al. (2008) for a review on ERPs. Using ERFs, Peyk et al. (2008) found greater activity for pleasant and unpleasant stimuli than neutral stimuli during the 120-170 window, with right positive polarity and left negative polarity. The source of this activity was located in occipito-parieto-temporal regions. D'Hondt et al. (2010) reported similar results at $180 \mathrm{~ms}$ after stimulus onset at occipito-temporal areas, and hypothesized that this activity could result from the rapid detection of emotional content by the amygdala.

Acknowledging that our analysis was performed at sensor level, our TF findings (Figure 4) can help us approximate the source location for activity occurring at around $170 \mathrm{~ms}$. Increased activity in the four frequency bands was located in both temporal regions, and the occipital region was the third one with greater activity. In particular, the temporal activity was most significant in the anterior superior sensors. Thus, our $170 \mathrm{com}-$ ponent presents certain similarities and certain differences with those found in previous facial processing and emotion studies: (a) activity is located in similar brain regions; (b) the location of the most significant values identified in our study were more anterior than those reported in the aforementioned literature; (c) the hemispheric distribution observed in our study is similar to Peyk et al. (2008), but opposite to that shown by other facial processing studies; (d) our analysis did not produce significant differences between conditions. How can we explain these similarities and differences?

We believe that points (a) and (b) support the notion that the M170 we found is related with perceptual and content processing. Note that our stimuli included no image portraying or suggesting faces. This might be why the activity resulting from our analysis is not located as posteriorly as that reported in face and emotion processing studies. It might be more anterior because of the semantic and content analysis involved in aesthetic appraisal. Another explanation could be related with task requirements. It is conceivable that deciding whether a picture portrays a face or a certain emotional expression or not requires processes that are more primitive than deciding whether one finds a stimuli to be beautiful or not. In this scenario, the second kind of task relies more on cognitive (frontal) or semantic (temporal) resources than the first kind. In relation with point $(\mathrm{d})$, decisions about beauty require a more refined and more precise processing beyond the first perceptual and cognitive stages, and the ERFs cannot detect it. Notice that the two next peaks (270 and 450$)$ had a more fronto-temporal distribution, which suggests a cognitive analysis rather than a perceptual one.

For several reasons, point (c) does not seem to be especially relevant. For instance, very few among the prior studies commented this issue. Moreover, there was no consistency among the studies as to the association between polarity and hemisphere. In any case, the main motivation of the present study was to characterize the dynamics of brain activity related with the appraisal of stimuli as beautiful and not beautiful, and this was the reason we carried out a TF analysis after the ERF analysis.

\section{TIME-FREQUENCY ANALYSIS}

In the first TF analysis we compared the baseline pre-stimulus power with the post-stimulus power. Significant differences appeared around $170 \mathrm{~ms}$ post-stimulus in the four frequency bands, and the pattern and power of oscillations were similar for beautiful and not beautiful stimuli (Figure 4). The largest difference between the baseline and the post-stimulus power appeared in the alpha frequency band. Alpha band seems to have a direct role in attention (Palva and Palva, 2007), working memory (Halgren et al., 2002), object recognition (Mima et al., 2001) and sensory awareness (von Stein et al., 2000). It is reasonable to believe that initial activity (up to $300 \mathrm{~ms}$ ) was related with some of these processes.

In the second TF analysis, we compared brain activity related with both kinds of stimuli. The comparison of the power associated with not beautiful stimuli against that associated with beautiful stimuli revealed no significant differences (note that $p<0.05$ for "Not Beautiful $>$ Beautiful" in Figure 5). The opposite contrast also produced no significant differences before $300 \mathrm{~ms}$, but it revealed that the oscillation power associated with beautiful stimuli was significantly greater than the power associated with not beautiful stimuli $400 \mathrm{~ms}$ after stimulus onset and beyond.

Lindsen et al. (2010) carried out a similar analysis with EEG data from a facial preference task using a 2AFC paradigm. Participants looked at one face until they decided to replace it with a second face, and then they indicated their preferred face in terms of approachability. Analysis of the power values showed that preferred faces presented in second place, but not those presented in first place, were related with an increased theta band activity around $500 \mathrm{~ms}$ over the fronto-central electrodes, and a decrease in the gamma band around $650 \mathrm{~ms}$ over central-occipital electrodes. These results in theta band in relation with the preferred second face are quite coincident with ours, though other results reported by Lindsen et al. (2010) are not. Although they used a face preference task and a very similar TF analysis to ours, a number of procedural differencesespecially the temporal arrangement of the stimuli and the type of decision, in addition to the important differences between 
registering brain activity with EEG and MEG_could account for different results.

In addition to revealing significantly greater power for beautiful stimuli than for not beautiful stimuli from $400 \mathrm{~ms}$ onwards, our results indicate that these differences occur in all four frequency bands. We believe that one possible interpretation of these results can be in terms of oscillation synchronization. The frequency of oscillations depends on cellular pacemaker mechanisms and neuronal network properties. Aesthetic appreciation, like many other cognitive faculties, emerges from the coordinated interaction of mechanisms and networks distributed across different brain areas (Nadal et al., 2008; Nadal and Pearce, 2011). The precise mechanisms underlying the coordination of these interactions - neural activity occurs at various spatial and temporal levels which must be dynamically adjusted-remains an unresolved problem in neuroscience (Uhlhaas and Singer, 2006). The synchronization of neural oscillatory activity constitutes a possible solution to this problem (Buzsáki and Draguhn, 2004; Schnitzler and Gross, 2005; Uhlhaas and Singer, 2006). Bhattacharya and Petsche $(2002,2005)$ have already highlighted the importance of synchronization in aesthetic tasks. Their analysis of EEG data showed significant differences in the degree of phase synchronization between artists and non-artists during visual perception of paintings (Bhattacharya and Petsche, 2002). Significantly higher synchrony was found in the high frequency beta and gamma bands in artists during the perception of the paintings. Since it has been claimed that these frequency bands are related with binding elementary visual attributes into a coherent ensemble, they interpreted their results as reflecting artist's enhanced ability for binding details of complex artworks to create internal representations.

In line with this argument, the greater oscillatory power observed in our study in relation with stimuli rated as beautiful might owe to a greater synchronization of oscillations. If this were the case, we could hypothesize that the general synchrony during the perception of beautiful stimuli would be the cause of the common effect across all bands. This interpretation, however, must be considered with caution, because, although a change in power spectrum often coincides with a change in synchronization, this is not always the case. Without losing sight of the exploratory nature of this study (and thus we used the FDR correction in the comparison tests), we believe it is interesting to ask what could the functional significance of this hypothesized synchronization while viewing beautiful images be? Could the frontal lobe be the hub of low frequency synchronization - theta and alpha?

Based on the Global Neuronal Workspace framework (Baars, 1993; Dehaene et al., 1998), as well as von Stein and Sarnthein (2000) and Palva and Palva (2007) proposals, our results might be seen as suggesting that a possible specific aesthetic global neuronal workspace is established during aesthetic tasks in which processing beautiful stimuli is related with a greater synchronization of neural activity than not beautiful stimuli. In this workspace for aesthetic appreciation, theta band activity would reflect the coordination and communication in several bands networks, and the control of working memory functions (Klimesch et al., 2010; Sauseng et al., 2010). Alpha band would reflect the internal, top-down processes, among others, expectations or generating hypothesis about the viewed stimuli. Gamma band would reflect local and basic visual analysis, like Gestalt principles or binding perceptual features (Nyhus and Curran, 2010). Finally, beta band would reflect semantic and supramodal binding related with the current stimulus.

\section{CONCLUSIONS}

The main objective of the present study was to describe the dynamics of brain activity during an aesthetic appreciation task. Thus, we carried out an ERF analysis and an exploratory TF analysis.

The ERF analysis revealed a peak of activity at about $170 \mathrm{~ms}$ independently of whether the stimulus was rated as beautiful or not beautiful. This M170 component was confirmed by the first $\mathrm{TF}$ analysis in which we compared activity before and after stimulus onset. This peak of activity originated in temporal regions. Previous studies have related the M170 with the beginning of the coding of object identity, and with the transformation of a sensory code to a cognitive processing, and with processes involved in resolving stimulus ambiguity. In this sense, we believe that the activity peak we observed at about $170 \mathrm{~ms}$ reflects this perceptualcognitive processing. Our ERF results, however, reveal no significant differences in activity related with stimuli considered beautiful and those considered not beautiful. Studies examining the neural underpinnings of emotion have found significant differences between ERFs related with neutral and non-neutral (pleasant or unpleasant) stimuli. Given the tight relation between pleasantness and beauty (Marty et al., 2003), we believe that there are no significant differences in our results because two non-neutral conditions were used. Thus, it seems appropriate to introduce the neutral response option in future experiments of aesthetic appreciation.

The TF analysis showed that oscillatory power related with beautiful stimuli was significantly greater than the power related with stimuli rated as not beautiful from 300-400 after stimulus onset, whereas the opposite contrast showed no significant differences. These differences appeared in the four frequency bands. Synchronization of oscillations could be a possible interpretation of those results. In earlier work (Nadal et al., 2008; Nadal and Pearce, 2011) we have argued, from evolutionary and cognitive points of view, that aesthetic appreciation emerges from the coordination of processes involving different brain regions. In light of the results presented in this paper, and in the absence of a firm candidate for the mechanism that explains such a coordinated interaction, we believe that future studies should test whether synchronization functions indeed as a coordination mechanism. Bhattacharya and Petsche $(2002,2005)$ results, in fact, revealed the importance of synchronization in tasks related with aesthetic appreciation. Although, as we have already noted, our interpretation must be considered with caution, our results could suggest that a specific aesthetic global neuronal workspace is configured during aesthetic tasks in which processing beautiful stimuli is related with a greater synchronization of neural activity than not beautiful stimuli. In this workspace for aesthetic appreciation, different frequency bands would reflect different perceptual and cognitive processes. Although this interpretation satisfies the need to account for the distributed spatial and temporal neural activity underlying aesthetic 
appreciation (Nadal et al., 2008; Nadal and Pearce, 2011), it is only based on the amplitude analysis and an exploratory experiment, which provides only a partial perspective of the synchronization. Additional synchronization analyses will be necessary to confirm our proposal (Bhattacharya and Petsche, 2005).

\section{REFERENCES}

Alegre, M., Labarga, A., Gurtubay, I. G., Iriarte, J., Malanda, A., and Artieda, J. (2003). Movement-related changes in cortical oscillatory activity in ballistic, sustained and negative movements. Exp. Brain Res. 148, 17-25.

Baars, B. J. (1993). A Cognitive Theory of Consciousness. New York, NY: Cambridge University Press.

Bar, M., and Neta, M. (2007). Visual elements of subjective preference modulate amygdala activation. Neuropsychologia 45, 2191-2200.

Bayram, M., and Baraniuk, R. G. (1996). Multiple window time frequency analysis. Proc. IEEE-SP Int. Symp. Time-Frequency Time-Scale Anal. 173-176.

Benjamini, Y., and Yekutieli, D. (2001). The control of the False Discovery Rate in multiple testing under dependency. Ann. Stat. 29, 1165-1188.

Bhattacharya, J., and Petsche, H. (2002). Shadows of artistry: cortical synchrony during perception and imagery of visual art. Brain Res. Cogn. Brain Res. 13, 179-186.

Bhattacharya, J., and Petsche, $\mathrm{H}$. (2005). Drawing on mind's canvas: differences in cortical integration patterns between artists and non-artists. Hum. Brain Mapp. 26, $1-14$.

Blood, A., and Zatorre, R. (2001). Intensely pleasurable responses to music correlate with activity in brain regions implicated in reward and emotion. Proc. Natl. Acad. Sci. U.S.A. 98, 11818-11823.

Blood, A., Zatorre, R., Bermudez, P., and Evans, A. (1999). Emotional responses to pleasant and unpleasant music correlate with activity in paralimbic brain regions. Nat. Neurosci. 2, 382-387.

Brown, S., Martinez, M., and Parsons, L. (2004). Passive music listening spontaneously engages limbic and paralimbic systems. Neuroreport 15, 2033-2037.

Buzsáki, G., and Draguhn, A. (2004). Neuronal oscillations in cortical networks. Science 304, 1926-1929.

Calvo-Merino, B., Jola, C., Glaser, D., and Haggard, P. (2008). Towards a sensorimotor aesthetics of performing art. Conscious. Cogn. 17, 911-922.
Calvo-Merino, B., Urgesi, C., Orgs, G., Aglioti, S., and Haggard, P. (2010). Extrastriate body area underlies aesthetic evaluation of body stimuli. Exp. Brain Res. 204, 447-456.

Campo, P., Poch, C., Parmentier, F. B., Moratti, S., Elsley, J. V., Castellanos, N. P., Ruiz-Vargas, J. M., del Pozo, F., and Maestú, F. (2010). Oscillatory activity in prefrontal and posterior regions during implicit letterlocation binding. Neuroimage 49, 2807-2815.

Castellanos, N. P., and Makarov, V. A. (2006). Recovering EEG brain signals: artifact suppression with wavelet enhanced independent component analysis. J. Neurosci. Methods 158, 300-312.

Castellanos, N. P., Paúl, N., Ordóñez, V. E., Demuynck, O., Bajo, R., Campo, P., Bilbao, A., Ortiz, T. del-Pozo, F., and Maestú, F. (2010). Reorganization of functional connectivity as a correlate of cognitive recovery in acquired brain injury. Brain 133(Pt. 8), 2365-2381.

Cela-Conde, C. J., Ayala, F. J., Munar, E., Maestú, F., Nadal, M., Capó, M. A., Del Río, D., López-Ibor, J., Ortiz, T., Mirasso, C., and Marty, G. (2009). Sex-related similarities and differences in the neural correlates of beauty. Proc. Natl. Acad. Sci. U.S.A. 106, 3847-3852.

Cela-Conde, C. J., Marty, G., Maestú, F. Ortiz, T., Munar, E., Fernandez, A., Roca, M., Rosselló, J., and Quesney, F. (2004). Activation of the prefrontal cortex in the human visual aesthetic perception. Proc. Natl. Acad. Sci. U.S.A. 101, 6321-6325.

Chatterjee, A. (2011). Neuroaesthetics: a coming of age story. J. Cogn. Neurosci. 23, 53-62.

Cupchik, G. C., Vartanian, O., Crawley, A., and Mikulis, D. J. (2009). Viewing artworks: contributions of cognitive control and perceptual facilitation to aesthetic experience. Brain Cogn. 70, 84-91.

D'Hondt, F., Lassonde, M., Collignon, O., Dubarry, A. S., Robert, M., Rigoulot, S., Honoré, J., Lepore, F., and Sequeira, H. (2010). Early brain-body impact of emotional arousal. Front. Hum. Neurosci. 4, 33. doi: 10.3389/fnhum.2010.00033

Dehaene, S., Kerszberg, M., and Changeux, J. P. (1998). A neuronal model of a global workspace in

\section{ACKNOWLEDGMENTS}

This research was supported by grant SEJ2007-64374/PSIC from the Spanish Ministerio de Educación y Ciencia. The authors are grateful to Almudena Capilla for helpful and useful comments on ERF analysis.

effortful cognitive tasks. Proc. Natl. Acad. Sci. U.S.A. 95, 14529-14534.

Gosselin, N., Peretz, I., Johnsen, E. and Adolphs, R. (2007). Amygdala damage impairs emotion recognition from music. Neuropsychologia 45, 236-244.

Grinsted, A., Moore, J. C., and Jevrejeva, S. (2004). Application of the cross wavelet transform and wavelet coherence to geophysicl time series. Nonlin. Process. Geophys. 11, 561-566.

Groppe, D. M., Urbach, T. P., and Kutas, M. (2011). Mass univariate analysis of event-related brain potentials/fields I: a critical tutorial review. Psychophysiology 48, 1711-1725.

Halgren, E., Boujon, C., Clarke, J., Wang, C., and Chauvel, P. (2002). Rapid distributed fronto-parietooccipital processing stages during working memory in humans. Cereb. Cortex 12, 710-728.

Halgren, E., Raij, T., Marinkovic, K. Jousmäki, V., and Hari, R. (2000). Cognitive response profile of the human fusiform face area as determined by MEG. Cereb. Cortex 10 , 69-81.

Itier, R. J., Herdman, A. T., George, N., Cheyne, D., and Taylor, M. J. (2006). Inversion and contrastreversal effects on face processing assessed by MEG. Brain Res. 1115 108-120.

Itier, R. J., and Taylor, M. J. (2002). Inversion and contrast polarity reversal affect both encoding and recognition processes of unfamiliar faces: a repetition study using ERPs. Neuroimage 15, 353-372.

Jacobsen, T., and Höfel, L. (2003) Descriptive and evaluative judgment processes: behavioral and electrophysiological indices of processing symmetry and aesthetics. Cogn. Affect. Behav. Neurosci. 3, 289-299.

Jacobsen, T., Schubotz, R. I., Höfel, L., and Cramon, D. Y. V. (2006). Brain correlates of aesthetic judgment of beauty. Neuroimage 29, 276-285.

Kawabata, H., and Zeki, S. (2004). Neural correlates of beauty. J. Neurophysiol. 91, 1699-1705.

Kirk, U., Skov, M., Christensen, M. S., and Nygaard, N. (2009a). Brain correlates of aesthetic expertise: a parametric fMRI study. Brain $\operatorname{Cog}$. 69 , 306-315.
Kirk, U., Skov, M., Hulme, O., Christensen, M. S., and Zeki, S. (2009b). Modulation of aesthetic value by semantic context: an fMRI study. Neuroimage 44, 1125-1132.

Klimesch, W., Freunberger, R., and Sauseng, P. (2010). Oscillatory mechanisms of process binding in memory. Neurosci. Biobehav. Rev. 34, 1002-1014.

Kloth, N., Dobel, C., Schweinberger, S. R., Zwitserlood, P., Bölte, J., and Junghöfer, M. (2006). Effects of personal familiarity on early neuromagnetic correlates of face perception. Eur. J. Neurosci. 24, 3317-3321.

Kobayashi, K., Jacobs, J., and Gotman, J. (2009). Detection of changes of high-frequency activity by statistical time-frequency analysis in epileptic spikes. Clin. Neurophysiol. 120 1070-1077.

Koelsch, S., Fritz, T., Von Cramon, D. Muller, K., and Friederici, A. (2006). Investigating emotion with music: an fMRI study. Hum. Brain Mapp. 27, 239-250

Lengger, P., Fischmeister, F., Leder, H., and Bauer, H. (2007). Functional neuroanatomy of the perception of modern art: a DC-EEG study on the influence of stylistic information on aesthetic experience. Brain Res. 1158, 93-102.

Lindsen, J. P., Jones, R., Shimojo, S., and Bhattacharya, J. (2010). Neural components underlying subjective preferential decision making. Neuroimage 50, 1626-1632.

Linkenkaer-Hansen, K., Palva, J. M. Sams, M., Hietanen, J. K., Aronen, H. J., and Ilmoniemi, R. J. (1998). Face-selective processing in human extrastriate cortex around $120 \mathrm{~ms}$ after stimulus onset revealed by magneto- and electroencephalography. Neurosci. Lett. 253, 147-150.

Liu, J., Harris, A., and Kanwisher, N. (2002). Stages of processing in face perception: an MEG study. Nat. Neurosci. 5, 910-916.

Liu, J., Higuchi, M., Marantz, A., and Kanwisher, N. (2000). The selectivity of the occipitotemporal M170 for faces. Neuroreport 11, 337-341.

Lovett, E. G., and Ropella, K. M. (1997). Time-frequency coherence analysis of atrial fibrillation termination during procainamide administration. Ann. Biomed. Eng. 25, 975-984. 
Lu, S. T., Hämäläinen, M. S., Hari, R., Ilmoniemi, R. J., Lounasmaa, O. V., Sams, M., and Vilkman, V. (1991). Seeing faces activates three separate areas outside the occipital visual cortex in man. Neuroscience $43,287-290$

Mallat, S. G. (1999). A Wavelet Tour of Signal Processing. New York: Academic Press.

Marty, G., Cela-Conde, C. J., Munar, E., Rosselló, J., Roca, M., and Escudero, J. T. (2003). Factorial dimension of aesthetic experience. Psicothema 15, 478-483.

Mima, T., Oluwatimilehin, T., Hiraoka, T., and Hallett, M. (2001). Transient interhemispheric neuronal synchrony correlates with object recognition. J. Neurosci. 21, 3942-3948.

Mitterschiffthaler, M., Fu, C., Dalton, J., Andrew, C., and Williams, S. (2007). A functional MRI study of happy and sad affective states induced by classical music. Hum. Brain Mapp. 28, 1150-1162.

Nadal, M., Munar, E., Capó, M. A., Rosselló, J., and Cela-Conde, C. J. (2008). Towards a framework for the study of the neural correlates of aesthetic preference. Spat. Vis. 21, 379-396.

Nadal, M., and Pearce, M. T. (2011). The Copenhagen Neuroaesthetics conference: prospects and pitfalls for an emerging field. Brain Cogn. $76,172-183$.

Nyhus, E., and Curran, T. (2010). Functional role of gamma and theta oscillations in episodic memory. Neurosci. Biobehav. Rev. 34, 1023-1035.

Olofsson, J. K., Nordin, S., Sequeira, H., and Polich, J. (2008). Affective picture processing: an integrative review of ERP findings. Biol. Psychol. 77, 247-265.

Palva, S., and Palva, J. M. (2007). New vistas for alpha-frequency band oscillations. Trends Neurosci. 30, 150-158.

Peyk, P., Schupp, H. T., Elbert, T., and Junghöfer, M. (2008). Emotion processing in the visual brain: a MEG analysis. Brain Topogr. 20, 205-215.

Quiroga, R., and Garcia, H. (2003). Single-trial event-related potentials with wavelet denoising. Clin. Neurophysiol. 114, 376-390.

Salimpoor, V., Benovoy, M., Larcher, K., Dagher, A., and Zatorre, R. (2011). Anatomically distinct dopamine release during anticipation and experience of peak emotion to music. Nat. Neurosci. 14, 257-262.

Sauseng, P., Griesmayr, B., Freunberger, R., and Klimesch, W. (2010). Control mechanisms in working memory: a possible function of EEG theta oscillations. Neurosci. Biobehav. Rev. 34, 1015-1022.

Schnitzler, A., and Gross, J. (2005). Normal and pathological oscillatory communication in the brain. Nat. Rev. Neurosci. 6, 285-296.

von Stein, A., Chiang, C., and König, P. (2000). Top-down processing mediated by interareal synchronization. Proc. Natl Acad. Sci. U.S.A. 97, 14748-14753. von Stein, A., and Sarnthein, J. (2000). Different frequencies for different scales of cortical integration: from local gamma to long range alpha/theta synchronization. Int. J. Psychophysiol. 38, 301-313.

Storey, J. D. (2002). A direct approach to false discovery rates. J. Roy. Stat. Soc. Ser. B 64, 479-498.

Susac, A., Ilmoniemi Risto, J., Pihko, E., Ranken, D., and Supek, S. (2010). Early cortical responses are sensitive to changes in face stimuli. Brain Res. 1346, 155-164.

Taylor, M., Itier, R., Allison, T., and Edmonds, G. (2001). Direction of gaze effects on early face processing: eyes-only versus full faces. Cogn. Brain Res. 10, 333-340.

de Tommaso, M., Pecoraro, C., Sardaro, M., Serpino, C., Lancioni, G., and Livrea, P. (2008). Influence of aesthetic perception on visual eventrelated potentials. Conscious. Cogn. 17, 933-945.

Torrence, C., and Compo, G. P. (1998). A practical guide to wavelet analysis. Bull. Am. Meteorol. Soc. 79, 61-78.

Uhlhaas, P. J., and Singer, W. (2006). Neural synchrony in brain disorders: relevance for cognitive dysfunctions and pathophysiology. Neuron 52, 155-168.

Vartanian, O., and Goel, V. (2004). Neuroanatomical correlates of aesthetic preference for paintings. Neuroreport 15, 893-897.

Verhoeven, K. J. F., Simonsen, K. L., and McIntyre, L. M. (2005). Implementing false discovery rate control: increasing your power. OIKOS 108, 643-647.

Xu, Y., Haykin, S., and Racine, R. J. (1999). Multiple window timefrequency distribution and coherence of EEG using Slepian sequences and hermite functions. IEEE Trans. Biomed. Eng. 46, 861-866.

Yue, X., Vessel, E. A., and Biederman, I. (2007). The neural basis of scene preferences. Neuroreport 18, 525-529.

Conflict of Interest Statement: The authors declare that the research was conducted in the absence of any commercial or financial relationships that could be construed as a potential conflict of interest.

Received: 31 March 2011; accepted: 21 December 2011; published online: 05 January 2012.

Citation: Munar E, Nadal M, Castellanos NP, Flexas A, Maestú F, Mirasso C and Cela-Conde CJ (2012) Aesthetic appreciation: event-related field and time-frequency analyses. Front. Hum. Neurosci. 5:185. doi: 10.3389/ fnhum.2011.00185

Copyright (C) 2012 Munar, Nadal, Castellanos, Flexas, Maestú, Mirasso and Cela-Conde. This is an openaccess article distributed under the terms of the Creative Commons Attribution Non Commercial License, which permits non-commercial use, distribution, and reproduction in other forums, provided the original authors and source are credited. 